\section{De la Casa de Orates al juzgado: pericia alienista y evaluación judicial de la locura en Santiago de Chile hacia 1860}

\section{From the Casa de Orates to the courtroom: psychiatric expertise and legal assessment of madness in Santiago de Chile around 1860}

\section{María José Correa Gómez}

Académica del Departamento de Artes y Humanidades/Universidad Andrés Bello. Quillota 910

2520000 - Viña del Mar - Chile

maria.correa@unab.cl
CORREA GÓMEZ, María José. De la Casa de Orates al juzgado: pericia alienista y evaluación judicial de la locura en Santiago de Chile hacia 1860. História, Ciências, Saúde - Manguinhos, Rio de Janeiro, v.20, n.2, abr.-jun. 2013, p.571-585.

\section{Resumen}

Este artículo explora el posicionamiento de la autoridad alienista en el ámbito de la justicia civil a través del estudio de evaluaciones periciales en procesos de interdicción por demencia en Santiago de Chile a mediados del siglo XIX. Presenta el marco legal que dio espacio a la figura del experto y se detiene en el ejercicio pericial cotidiano, con el objeto de abordar algunas de las fuerzas que legitimaron su autoridad en las cambiantes condiciones del temprano alienismo.

Palabras clave: locura; interdicción; alienismo; pericia; Santiago de Chile.

\section{Abstract}

This article explores the position of mental health authorities in the civil justice field by studying psychiatric evaluations in cases of judicial interdiction on account of dementia in Santiago de Chile in the mid nineteenth century. It examines the legal framework that created a space for the figure of the expert and focuses on the everyday use of psychiatric evaluations, in order to explore some of the forces which legitimized their authority in the changing conditions of early psychiatry.

Keywords: madness; interdiction; psychiatry; expert testimony; Santiago de Chile. 
$I^{1}$ nvestigadores como David Wright caracterizaron el proceso de certificación de la locura, es decir el momento de identificación, interpretación y atestación médica de una enfermedad mental, como una instancia central en la formación y difusión de las ideas psiquiátricas y del carácter profesional y experto del contingente médico (Wright, 1998). Considerando dicha centralidad, este estudio atiende al posicionamiento del perito alienista, a la negociación de su identidad profesional y a la defensa de su habilidad para identificar comportamientos 'desviados' y diagnosticar estados patológicos entre una variedad de expertos que participaban en procesos judiciales tales como médicos generales, de familia y de ciudad. Adicionalmente se detiene en el desarrollo de la autoridad del perito y en las herramientas interpretativas usadas para negociar los significados de la locura hacia mediados del siglo XIX chileno. La contextualización de este ejercicio revela parte de las circunstancias que llevaron a la formación de especialistas en el área de la insania y su relación con cambios más generales, experimentados durante la segunda mitad del siglo XIX, como la creación del primer asilo para el tratamiento de la locura (1852) y los procesos de especialización médica.

Catherine Crawford (1993) consideró a la historia de la medicina forense como parte integral de los proyectos de medicalización, toda vez que instaló la autoridad médica en nuevos territorios sociales, como el de la justicia. En este sentido, la certificación judicial posibilita explorar la legitimación del facultativo en tanto experto forense y por ende promueve nuevos ángulos de análisis basados en la negociación entre Estado, derecho y medicina. ${ }^{1}$ En Chile, la propagación de los conocimientos específicos manejados por los facultativos y su validación como testigos expertos fueron resultado de una interacción similar, en un contexto marcado por el deseo de especialización que aplaudía la aplicación de competencias específicas en la labor pericial.

Este artículo se detiene en el estudio de estas competencias por medio del análisis de la práctica pericial cotidiana y de las evaluaciones emitidas por testigos expertos en casos de interdicción. El estudio considera un total de 75 procesos de interdicción presentados ante el Juzgado Civil de Santiago entre 1855 y 1900. Estos llevaron a la realización de 120 diligencias periciales que resultaron en el envío de 78 informes escritos (individuales y colectivos) en los que participaron 45 médicos, varios de ellos, en particular los alienistas, en un número repetido de veces.

A través del estudio de estos procesos judiciales y las instancias de certificación médica se problematizará la extensión de las fronteras de la ciencia médica y en particular de la temprana autoridad alienista en la sociedad chilena de la segunda mitad del siglo XIX. ${ }^{2}$ El análisis se concentrará en un proceso judicial, insistiendo en la riqueza interpretativa del proceso en su conjunto como un momento que presenta adicionalmente la relación médico-paciente o demandante-testigo-demandado y sus vínculos con las condiciones de realización y recepción de la pericia. Los lazos establecidos entre el perito y las partes, sus pares y los representantes de la justicia fueron fundamentales para que personas no expertas requirieran, interactuaran o rechazaran la mirada del especialista, develando en el ejercicio cotidiano pericial los contextos particulares que levantaron o erosionaron la verosimilitud de la opinión y diagnóstico médico.

Resulta significativo explorar la evaluación médico legal en tanto revela, siguiendo la crítica epistemológica feminista, ciertas problemáticas asociadas a los modos de construcción 
y legitimación de la 'verdad científica' (Williams, 1998). En este sentido, preguntarse por la incorporación del 'experto' a un determinado escenario, la forma en que observó y entregó 'su ciencia' y como ésta fue validada o rechazada, resulta central para explorar la pluralidad de bases que nutrieron la constitución de dichas 'autoridades' y de sus 'verdades'. En el caso particular de la pericia médica, se vuelve necesario reflexionar sobre el reconocimiento de su autoridad y sobre la legitimación de los procesos de construcción de veracidad para acceder no solo a los mecanismos de validación, sino también a los matices y texturas de la autoridad y del conocimiento en su ejercicio cotidiano.

\section{Notas sobre la interdicción por demencia}

La ley sobre la Casa de Orates (1856) constituyó la primera regulación republicana en ordenar la guarda de dementes en instituciones médicas, mientras que los artículos sobre interdicción presentados en el Código Civil (1855) reformularon la curatela colonial, delineando las nuevas condiciones para la restricción de la capacidad de disipadores, sordomudos y dementes. Ambas prácticas nutrieron un proceso de examinación y certificación de la locura, el cual extendido en el tiempo contribuyó al posicionamiento de los médicos - en particular los alienistas - como expertos en la identificación y entendimiento de las enfermedades mentales (Correa Gómez, 2012). ${ }^{3}$

La interdicción por demencia correspondió al estado de una persona a quien por sentencia de término se le declaraba incapaz de los actos de la vida civil y se lo sometía a curatela (De La Plata, 1887). Esta sentencia acarreaba importantes consecuencias, entre ellas la pérdida de la capacidad jurídica, es decir, la facultad de disfrutar derechos y estar sujeto a deberes, anulando todos los actos y contratos realizados con posterioridad al juicio. Esta muerte cívica resultaba de una contienda legal originada del deseo o necesidad de despojar al demandado de la administración de sus bienes o de los que podría recibir en el futuro. ${ }^{4}$ La interdicción debía ser solicitada por parientes, pero si el insano carecía de familia, representaba un peligro para la comunidad o ponía en riesgo su propia vida, podía ser presentada por vecinos o compañeros de trabajo, así como por defensores públicos y funcionarios diplomáticos o consulares (Amunátegui, 1865).

El proceso judicial se centraba en la confirmación de la incompetencia. Para ello, el juez debía recabar información sobre la vida y comportamiento del supuesto demente a través de testimonios de amigos, vecinos o familiares y oír el dictamen de facultativos, quienes debían confirmar la existencia y naturaleza de su condición. El Código Civil no estipuló los conocimientos que debían manejar dichos facultativos ni tampoco las características de la evaluación; por el contrario, solo indicó que debían ser de la confianza del juez (Correa Gómez, 2009). ${ }^{5}$

Tras la confirmación de la supuesta demencia se coordinaba la curatela del interdicto, la que debía ser administrada preferentemente por parientes y a falta de candidatos por conocidos o abogados. El establecimiento de dicha guarda confirmaba el estado de completa incompetencia del demente y supuestamente establecía una estructura de protección, cuidado y vigilancia sobre aquellos que carecían ante la ley de la aptitud mental para administrar sus vidas y sus bienes. Como revelan los juicios de interdicción, la curatela se articuló no solo por el deseo 
de protección, sino también y en un grado no menor, por la ambición de controlar los bienes del insano, motivando conflictos familiares y problemas en las curadurías. La influencia del tutor no se circunscribía solo al manejo de contratos legales, sino a la administración de aspectos cotidianos, como los tratamientos terapéuticos, la alimentación o la vestimenta. Este debía manejar los recursos de una manera conveniente, responder por su conservación y usarlos para enfrentar los gastos que implicaba la enfermedad del incapacitado (Amunátegui, 1865; Del Real, 1903). La ley aceptaba el nombramiento de dos guardianes, uno dedicado al manejo de los recursos y otro al cuidado de la persona del insano. Sin embargo, esta última actividad no podía ser realizada por quienes podían heredarlo, salvo el padre, la madre o alguno de los esposos. El proceso obligaba la inscripción de la interdicción en el Registro del conservador y su notificación al público por medio del periódico del departamento y carteles exhibidos en lugares concurridos.

El estudio en profundidad del proceso judicial de interdicción permite observar otro ángulo del proyecto de medicalización de la sociedad chilena, detenerse en ciertos actores y en sus relaciones con las circunstancias cotidianas de la medicina en curso. Permite revisar la gradual instalación de la ciencia médica y del saber alienista en los espacios judiciales, así como, también, profundizar en las tensiones que acompañaron este proceso, marcadas por la influencia de hombres y mujeres ajenas a las esferas del conocimiento formal en la modelación y aplicación del saber experto. ${ }^{6}$

\section{Notas sobre la formulación legal de la pericia médica}

El estudio de la práctica judicial ha dado cuenta de la larga data de facultativos como agentes de un conocimiento especial en procesos criminales y civiles. ${ }^{7}$ A pesar de que, en Chile, su participación como testigos se remonta al periodo colonial, no fue sino hasta la sistematización de la ley republicana que su carácter probatorio, al ser formalizado y por tanto exigido, fue adquiriendo peso. Este posicionamiento respondió en gran medida a dos procesos significativos: la medicalización de la sociedad chilena y el ingreso del alienismo y la gradual judicialización de los conflictos y relaciones sociales. La primera llevó a la regulación de la práctica médica, al desarrollo de políticas de salud pública y al consiguiente reforzamiento de la autoridad de la disciplina en la sociedad. La segunda respondió a cambios expresados en el ámbito de la justicia, donde nuevos ordenamientos buscaron regular el ejercicio judicial y asegurar en ella testimonios confiables y veraces; entre ellos, las declaraciones realizadas por quienes manejaban un determinado arte o ciencia.

La legitimación teórica de la pericia respondió al proceso de profesionalización de la salud, el cual se articuló desde diversos frentes marcados, como propuso Morrell (1996) para el caso europeo, por el desarrollo de trabajos de tiempo completo y mejor remunerados, la obtención de calificaciones especiales, la creación de nuevos procedimientos de formación, el surgimiento de un circuito editorial, la gestación de una identidad grupal, así como el reconocimiento de los buenos hábitos disciplinarios. Un marco similar se perfiló en Chile durante la segunda mitad del siglo XIX, delineado por la ampliación de las labores médicas en instituciones públicas, como la Casa de Orates, la extensión de la malla curricular de los estudios médicos, el envío de jóvenes facultativos a perfeccionarse a Europa, el surgimiento de 
revistas especializadas, la formación de agrupaciones científicas y los concursos desarrollados al alero de estas para premiar y legitimar investigaciones y trayectorias.

La historiografía chilena ha subrayado y explorado el rol del Estado en este proceso por sobre otros contextos ligados a la especialización y al quehacer médico cotidiano. Sol Serrano, por ejemplo, reconoció en la formación, aceptación y consumo de una base cognitiva certificada las principales condiciones que llevaron a la profesionalización del saber médico y al Estado como propulsor de estas bases y de los mecanismos de protección de la exclusividad médica (Serrano, 1994). En una línea similar, Murdock (1995) planteó una marcada intervención estatal en el ejercicio de la actividad médica, la cual incidió en sus propias estrategias de profesionalización. ${ }^{8}$ Adicionalmente, estas medidas otorgaron espacio para el desarrollo de nuevas áreas de saber, así como ayudaron a levantar el estatus científico de la medicina a través de su vínculo con el aparato estatal. ${ }^{9}$

Efectivamente, vemos como durante el transcurso del siglo se gestaron una serie de regulaciones que defendieron la profesionalización médica. Enfatizando la importancia de la certificación, la ley de 1842 impidió el ejercicio de la profesión científica a quienes no contasen con el grado de licenciado conferido por la universidad, marginalizando de ciertos circuitos a un número importante de practicantes, como estudiantes retirados de la carrera de medicina, parteras y flebótomos. ${ }^{10}$ Así, la injerencia estatal abarcó desde la formación universitaria ${ }^{11}$ hasta aspectos relacionados con la ética médica (como el secreto profesional), los cuales fueron debatidos constantemente desde distintos órganos, tales como la Sociedad Médica de Santiago (1869) y su canal de expresión, la Revista Médica (1872).

En la esfera judicial, la ley indagó en la figura del perito y gradualmente esbozó sus responsabilidades. Los cambios destinados a agilizar los procedimientos dieron cuenta de la progresiva importancia del experto tanto en la verificación del cuerpo del delito como en la evaluación de la responsabilidad criminal, paralelo a la incorporación del marco positivista. Con el tiempo, el testimonio médico fue cobrando valor, disminuyendo el número de historias como la del capitán norteamericano Henry Paddock, ejecutado a inicios de 1833 por orden del gobernador militar, Diego Portales, tras asesinar a algunos hombres en el puerto de Valparaíso y pese a que el doctor Guillermo Blest, entre otros, defendió su irresponsabilidad aduciendo locura (Costa, 1984). Atrás también parecían quedar las palabras de Portales, su celo por las leyes y la poca misericordia mostrada hacia quienes actuaban movidos por la "aberración mental", "puede muy bien suceder que Paddock padeciese alguna aberración mental ... si le justificamos dando valor a su excepción de insania, no habrá ya quien no quede impune de un crimen alegando la misma excepción ... No duden ustedes que en lo sucesivo si Paddock salva la vida, la excepción de insania va a sustituir a la de embriaguez" (De la Cruz, 1937, p.332).

En el ámbito de la justicia civil, el Código Civil de 1855 - puesto en marcha en 1857 instauró la obligatoriedad de la información pericial en procesos de interdicción, contrastando con la irregular presencia médica en la curatela de dementes. ${ }^{12}$ Ahora bien, esta imposición contribuyó a abrir el debate en torno a la fuerza probatoria de los peritos y a la relación establecida entre jueces y médicos. El Prontuario Judicial estipuló, paralelo al cuerpo de leyes civiles, que en los casos en que se requirieran conocimientos especiales, podía dudarse si los jueces estaban o no obligados a conformarse con el dictamen de los peritos (Vila, 1857). 
El Código Penal (1874) sancionó a los expertos que se negaban a prestar declaración o a "practicar una operación propia de su oficio" (Vera, 1884, p.65; Puga Borne, 1896, p.48). ${ }^{13}$ En este contexto, los médicos se encontraban teóricamente bajo la autoridad de la justicia, obligados a realizar la evaluación y a emitir informes una vez requeridos por el juez tanto en el ámbito penal como civil, los que a su vez podían ser cuestionados y contrastados. Se exhibía por parte del discurso legal una opinión ambivalente del saber pericial, sustentado por un lado en la consideración de los médicos como los más indicados para identificar la locura, discernir si era permanente o temporal o si se estaba frente a un caso de simulación. Por otro lado, se expresaba un temor a los informes poco escrupulosos, dado por empeños o por un examen poco prolijo, que podían inculpar "de un crimen" o penalizar a individuos inocentes, así también salvar a criminales (Vera, 1884, p.90).

Por cierto, subrayando las graves consecuencias de una evaluación mal realizada, el Prontuario sugirió tres vías a considerar en la identificación de la locura: la información sobre el comportamiento cotidiano del enfermo mental, los posibles rasgos o características que denotaran su condición (como, por ejemplo, su caligrafía) y la interrogación (Vera, 1884, p.278-279). Pese al reconocimiento de estas vías periciales, el Código de Procedimiento Civil (1903) no estableció las condiciones del conocimiento experto, no ahondó sobre los significados específicos de la demencia ni la locura, ni esbozó las características del reporte y del saber que debía entregar y manejar el experto en procesos de interdicción. Esta situación incidió sobre la autoridad del perito, más aún si consideramos que el cuerpo legal señaló que la fuerza probatoria del reporte forense debía ser considerada por el juez como una presunción, dependiente de la competencia de los expertos y del acuerdo o desacuerdo en las opiniones y principios científicos (Toro y Echeverría, 1902, art. 501).

Así, el siglo XX se iniciaba con la pervivencia de una lógica que reunía la legitimación del sentido común de los jueces y la expansión de la libertad en la interpretación de la ley (Stabili, 2000), pero también con conocimientos y procesos más formales en torno a los significados de la locura y su identificación pericial. En este escenario, los médicos, con todas sus contradicciones, se habían transformado en agentes centrales de la interpretación judicial de la locura, pese a la demora de la ley en graficar su competencia específica para la interpretación judicial de la capacidad mental.

Los médicos defendieron su capacidad de identificar la sanidad de la locura y, en este contexto, respondieron a las incursiones del Estado y del derecho en su disciplina preocupándose de destacar su autoridad en los reconocimientos judiciales y de enfatizar la distancia que separaba a los peritos de los testigos ordinarios. El perito, a diferencia del testigo, señalaba la Revista Médica, órgano representativo de la Sociedad Médica, debía tener "ciertas condiciones de idoneidad, de capacidad para dilucidar las cuestiones que se le someten porque siempre obra profesionalmente y de un modo general". El testigo, en cambio, solo refería los hechos presenciados, "pudiendo ser tal, todos los individuos que tengan solamente sentido común para poder percibir los hechos" (Schneider, 1872, p.123).

La actividad pericial en procesos de interdicción se expresó en este marco de formalización y definición del rol del experto. Este ejercicio fue realizado por los médicos convocados por los jueces, aquellos sugeridos por los demandantes o demandados y aceptados o rechazados por las autoridades, así como por los facultativos que participaron como testigos comunes 
de imputados institucionalizados en hospitales, hospicios o en la Casa de Orates. En estas instancias encontramos una pugna de saberes y una dificultad de identificar, evaluar y comunicar la demencia que pensamos no sólo fue constitutiva a la formación de la especialización y de la autoridad alienista, sino también a las normativas que regularon su accionar en un tiempo en que las cambiantes percepciones en torno a la enfermedad mental desafiaban y dificultaban su manejo judicial.

\section{De la identificación al diagnóstico: monomanía y temprano protagonismo alienista}

En septiembre de 1862, Francisco Ruiz Tagle solicitó la interdicción por demencia de su esposa Joaquina Aldunate, en el contexto de una pugna judicial extendida por más de cinco años - que incluyó demandas por separación, administración y restitución de bienes y cuidado de los hijos. Su petición se basaba en las extravagantes ideas que martirizaban a su esposa en el último tiempo y en el carácter hostil que mostraba a su familia, situaciones que se contraponían a los dieciocho años que la pareja había vivido, en palabras de Francisco, en la más perfecta inteligencia y unión.

Los testigos de la parte demandante, todos ellos representantes de la elite capitalina, coincidieron en presentar el comportamiento errático que mostraba Joaquina desde la muerte de su hijo mayor - fallecido tras caer desde un balcón en el Seminario Conciliar del Arzobispado - en términos de una perturbación mental. Sor Clara, de la orden San Vicente de Paul, indicó que las pocas veces que Joaquina asistió al hospital para "servir a los enfermos" solía ponerse a llorar manifestando lo infeliz que era y la falta de recursos a la que la exponía su marido, "soltando luego la risa con facilidad". Explicó también que ésta le había ofrecido remedios homeopáticos y que al ver a un religioso que aborrecía manifestó su malestar "con ademanes propios de una loca". Una amiga de la pareja, Gertrudis Montes, sostuvo que Joaquina divagaba en sus conversaciones, hasta el punto de decir que había tenido revelaciones y hablado con el mismo dios; mientras el sacerdote y futuro obispo, Joaquín Larraín Gandarillas, insistió en que desde hacia un año que sus palabras no hacían más que acusar la perturbación de su razón (Interdicción, 1862, f.3-5).

Francisco precisó que había hecho todo lo posible por silenciar la triste enfermedad de su esposa, sin embargo, agotados los esfuerzos y bajo la necesidad de cuidar la reputación y los bienes de Joaquina, decidió iniciar el proceso. Su preocupación por exponer conflictos domésticos, cruzar el umbral de lo privado e instalar la insania en el ámbito de la justicia fue compartida por quienes requirieron la interdicción de sus cercanos. En general, un procedimiento judicial de este tipo implicaba el destape de una serie de tensiones privadas que transitaban por la estructura familiar, exacerbadas por los desajustes que ocasionaba el comportamiento insano o, algunas veces, solo anárquico de un pariente. Los demandantes incorporaron expresiones de dolor y vergüenza por las implicancias de restringir la autoridad de padres, madres y abuelos o de limitar la capacidad de hermanos y hermanas; así, también, palabras de desazón por la pérdida de la confianza en la recuperación del demente. La interdicción, a diferencia de la internación en la Casa de Orates, implicaba un cambio legal en el estado de una persona y no una reclusión terapéutica que tendió a ser transitoria. Esto explica el tiempo transcurrido entre el inicio de la demencia y la petición de interdicción, la 
reserva de algunos demandantes y el hecho de que la gran mayoría de las demandas naciera de una circunstancia específica que agudizó el conflicto que generaba la demencia e instaló a la privación de las libertades civiles como la solución más adecuada.

Iniciado el proceso y con la información provista por algunos de los muchos testigos que participaron del caso, el juez requirió la mirada experta de la medicina y sus representantes: médicos titulados en instituciones extranjeras o en la Universidad de Chile. Ahora bien, la evaluación de la comisión médica, formada por Lorenzo Sazié (1807-1865), José Ramón Elguero (1819-1877) y Antonio Torres, difirió de las descripciones de amigos y conocidos de la familia. Tras tres examinaciones y en un informe de 4 páginas indicaron que Joaquina no se encontraba demente y pese a que su avanzado grado de obesidad, temperamento sanguíneo y susceptibilidad nerviosa, favorecían la agitación y explicaban su abatimiento moral, ella entregaba respuestas precisas sobre su familia y sobre sus intereses (Interdicción, 1862, f.31-32).

El defensor de menores enfatizó que el informe experto debía ser más atendible que la visión de los testigos legos y las pruebas entregadas por el demandante - un grupo de confusas cartas enviadas por Joaquina a un sacerdote lazarista - y propuso no aceptar la interdicción. En este contexto, Francisco insistió en la emisión de un nuevo informe y argumentó, desplegando un cierto manejo del ideario alienista, que los médicos probablemente habían visitado a Joaquina en un "estado tranquilo" (Interdicción, 1862, f.33-33v). Tomando esta petición, el juzgado notificó a Elguero y Torres, sumando a Estalisnao Ríos como remplazante de Sazié. Tras realizar un examen físico y considerar todo aquello que pudiera perturbar las funciones cerebrales, los médicos concluyeron que existía en ella una "aberración mental" calificada, "según Esquirol, de monomanía razonante". Estas circunstancias la colocaban en la "triste situación de una monomaníaca" y la inhabilitaban, por tanto, para la administración de su persona y de sus bienes (Interdicción, 1862, f.37).

La dificultad en la identificación de la locura se caracterizó no solo por la distancia entre la apreciación lega y experta, sino principalmente por la divergencia médica. Los juicios de interdicción proyectan la negociación de saberes, los esfuerzos interpretativos y las complejidades del uso de un cuerpo teórico y de un método de diagnóstico, a diferencia del mayor grado de consenso desplegado por los estudios y casos clínicos decimonónicos presentados en revistas académicas como la Revista Médica o los Anales de la Universidad de Chile.

La contradicción de los dos reportes llevó al defensor de menores a solicitar una nueva instancia pericial para evaluar a Joaquina, "no una, sino las veces que fuera necesario para dar un informe reposado y concienzudo" (Interdicción, 1862, f.38). Sin embargo, los doctores Juan Macdermont, Vicente Padín y Jorge Petit no lograron entregar una respuesta concluyente, pues pese a que las transiciones de llanto y risa de Joaquina, las cartas, los testimonios y la insistencia de la imputada sobre la pérdida de su patrimonio sugerían la presencia de un desarreglo, no se detectaron indicios claros que confirmaran un trastorno.

Nuevamente la respuesta no cumplió las expectativas de la justicia y así, tras tres meses de iniciado el caso y la participación de varios facultativos, la capacidad de Joaquina resultaba aún incierta. La locura no era una condición simple de certificar, tampoco de comunicar. Ahora bien, pese a que estas dificultades se expresaron en gran número de procesos de incapacidad, no impidieron el posicionamiento de la autoridad médica ni el reconocimiento del especialista. 
Los médicos llamados a evaluar a Joaquina formaron parte de una elite relacionada con la Universidad de Chile. El francés Lorenzo Sazié, contratado por el gobierno en 1833, había sido el primer decano de la escuela y, para 1862, se desempeñaba como profesor de clínica externa y obstetricia y como médico jefe de la Casa de Orates; Jorge Petit, también francés, había llegado a Chile en 1848 y dictaba clases de clínica interna e higiene; Vicente Padín enseñaba fisiología y medicina legal y al año siguiente de su participación en el juicio sería nombrado decano de la Escuela de Medicina de la universidad; Antonio Torres, español educado en la escuela portuguesa, había arribado a Chile en 1818 e ingresado como miembro de la Escuela de Medicina en 1848; mientras que José Ramón Elguero, principal representante de la ciencia alienista que comenzaba a tomar forma en ese periodo, además de desempeñarse como médico de la Casa de Orates (1860-1874), era académico de la universidad desde 1854 y formador de futuros alienistas a través del loquerío y de un breve curso sobre enfermedades mentales que se desarrollaría entre los años 1869 y $1870 .{ }^{14}$

Estos evaluadores integraron diferentes conocimientos y exhibieron una autoridad sustentada en su labor docente, en su posicionamiento institucional y en la práctica cotidiana. En este contexto, la Universidad de Chile y la Casa de Orates de Santiago fueron instituciones centrales al proceso de legitimación de la opinión médica y a la transformación de los llamados 'discursos de verdad', problematizados por Foucault (2003) para el contexto francés. Ambas instituciones respaldaron el posicionamiento de los primeros expertos y la interpretación realizada de ciertos cuerpos y comportamientos como 'dementes'.

En este contexto, Joaquina fue diagnosticada sufriendo de monomanía, condición descrita en Francia, a inicios del siglo XIX, por Jean Etienne Dominique Esquirol (1782-1840) que aludía principalmente a una idea fija que se imprimía en el enfermo y que contaminaba la sanidad de su mente. En su artículo "Monomanía", de 1818, Esquirol la presentó como una entidad patológica ubicada en una posición intermedia entre la manía y la lipemanía, forjada al alero de la renovación del sistema de clasificación que se llevaba a cabo en ese periodo (Goldstein, 2001). La monomanía, en términos más amplios, hacía referencia a la locura parcial, a un desorden en las facultades del individuo que no se relacionaban con problemas en el intelecto. Esto suponía un desafío para todos quiénes debían establecer un diagnóstico, sea médico o social, dadas las características propias de una condición que no se develaba con facilidad.

En el caso de Joaquina, los testigos que compartieron la visión de Francisco detallaron en su lenguaje lego que había momentos en que su conversación estaba en "el mejor estado de lucimiento" y momentos en que "divagaba de un modo extraño", que solía tener "intervalos en que su carácter era intolerable y obstinado" y que su locura "se notaba más en las relaciones domésticas y de confianza". Algunos corroboraron la presencia de ciertas ideas fijas como el odio a algunos religiosos y la insistencia con que acusaba su falta de recursos, mientras otros consideraron "que tenía la razón extraviada (por intervalos)" y "que sufría alguna enfermedad mental, aunque parcial", pues contestaba con exactitud y acierto muchas de las preguntas. (Interdicción, 1862, f.2v-3).

La relación entre lucidez y locura alertaba a los abogados, complicaba a demandantes, ponía en riesgo a demandados y desafiaba la evaluación experta. Si bien los testigos legos coincidieron en afirmar que Joaquina tenía algún grado de desorden en sus facultades, las características propias de su aparente locura parcial confundían al observador, abriendo un 
espacio para la duda, las contradicciones y la subjetividad en la construcción de la verdad. La oposición entre los dos primeros informes de Elguero pareció ser entendido por los médicos como parte de la dificultad propia del ejercicio de su especialidad, como problemática que acompañaba la preparación de unos pocos expertos y como respuesta a los desafíos de las locuras parciales, el intervalo lúcido y particularmente, la monomanía.

La historiografía europea y norteamericana ha considerado a la monomanía como una de las condiciones que más claramente corporalizó y congregó los cambios expresados durante el siglo XIX en torno al entendimiento de la locura. Consideró la gestación de esta categoría como una instancia que metaforizó los cambios en el modo de entender el ejercicio médico, la terapéutica y la especialidad profesional en torno a la insania, toda vez que no aludía a una demencia absoluta, sino más bien a personas "de inteligencia" sanas que movidas por ideas fijas, pasiones y emociones, realizaban actos irracionales, algunos incluso criminales. Como Jan Goldstein ha planteado, esta renovación de la nomenclatura médica, y su relación con los profundos cambios vividos en la sociedad francesa de inicios del siglo XIX, se vincularon al posicionamiento de la autoridad y del prestigio del alienismo y sus representantes (Goldstein, 2001, p.158). En una perspectiva similar y situándonos en el contexto chileno, es posible sugerir que el manejo de nuevas categorías en la justicia y la ampliación de la taxonomía diagnóstica incidieron no solo en aspectos generales de medicalización de la locura, sino particularmente sobre la apreciación de la pericia alienista.

En la historia de Joaquina, la conceptualización de la locura parcial bajo el diagnóstico de monomanía fue clave para decretar su interdicción. ${ }^{15}$ Esta etiqueta permitió la contradicción y la negociación del diagnóstico médico, validando la pericia alienista. De este modo, el reconocimiento de perturbaciones difíciles de precisar y que no siempre comprometían el intelecto, como la monomanía razonante de Esquirol, reforzó la autoridad de un grupo de especialistas médicos que buscaba posicionar su naciente autoridad por medio de su capacidad para reconocer la veracidad o falsedad de la locura imputada.

Los diagnósticos específicos presentados por el alienista José Ramón Elguero, durante la década del 1860 en causas de interdicción, no eran del todo nuevos. El uso de la monomanía se vinculó a un contexto en el cual los primeros especialistas comenzaron a emplear denominaciones más específicas para nombrar condiciones que tendían a describirse con anterioridad en términos de fatuidad y completa demencia, entre varios otros. El tránsito y adaptación de categorías conceptualizadas en las escuelas europeas no se hizo esperar y ya hacia mediados del siglo, paralelo a la formación de la Casa de Orates, encontramos diagnósticos periciales que dan cuenta de un variado cuerpo de terminologías en circulación que hacía alusión a condiciones congénitas como el cretinismo, la imbecilidad y la debilidad de espíritu, así como a problemas mentales adquiridos como la histeria, el delirium tremens, la lipemanía, la melancolía, la meningitis y la parálisis general progresiva, entre otros. En 1834 encontramos, por ejemplo, uno de los primeros casos de incapacitación por monomanía, diagnosticado por los profesores de la Escuela de Medicina Juan Miquel y Pedro Morán, mientras que décadas más tarde, y para el año en que se desarrollaba el juicio de Joaquina, un 11\% de los ingresados a Casa de Orates fueron identificados como monomaníacos (Elguero, 1863, p.175). ${ }^{16}$

Ahora bien, podemos proponer que dicha presencia se amplificó a través de la certificación de la locura y la presentación de diagnósticos en el ámbito judicial proyectando los marcos 
interpretativos utilizados, primero en la Universidad y luego en la Casa de Orates, hacia los juzgados y las calles de Santiago. Las evaluaciones periciales, realizadas por José Ramón Elguero, revelan la puesta en uso de un conocimiento que, como advertía el propio alienista en un estudio presentado ante el Gobierno en 1863, recién comenzaba a tomar forma. "He concluido mi tarea con la justa desconfianza de no haberla desempeñado como lo requiere la ciencia y mis deseos; infinitos vacíos notaréis en ella, pero a vuestra indulgencia unid esta consideración: que es el primer trabajo de esta especie que se hace en nuestro país y que yo, sin preparación práctica para el tratamiento de esta clase de enfermedades, he tenido el arrojo de emprenderlo, no me quejo, pues la suerte me ha sido propicia, y en más de una vez he logrado devolver la razón a algunos desgraciados. Tened presente que en nuestra escuela médica no ha habido ni hay clínica de estas dolencias" (Elguero, 1863, p.187).

Hacia 1860 el reconocimiento de la temprana autoridad alienista resultaba complejo, no solo por las características propias de la insania, sino también por el sistema formativo de los primeros alienistas. Es de suponer que en un contexto en el cual no circulaban mayores estudios locales sobre enfermedades mentales, ni tampoco revistas especializadas de circulación periódica, la certificación de la locura en el ámbito judicial representó un importante espacio de demostración de saber y autoridad. Por esto, pese a que la ley no obligaba a realizar diagnósticos en los procesos de interdicción sino solo a certificar la existencia de una perturbación e indicar si imposibilitaba la administración de los bienes, podemos pensar que los peritos, en particular aquellos considerados expertos en enfermedades mentales, comenzaron a incorporar en sus evaluaciones la nomenclatura que daba cuerpo y especificidad a su ciencia con la idea de posicionar su autoridad. Elguero (1863, p.174) representó un claro ejemplo de ello, pues no se limitó a certificar una incapacidad, sino que exhibió el manejo de las voces técnicas del alienismo contemporáneo, que él mismo presentaría en su estudio de 1863, y dejó entrever en algunos casos las posibilidades terapéuticas ofrecidas por su especialidad. ${ }^{17}$

Los reportes médicos emanados de procesos de interdicción muestran que hacia 1860, y en una primera etapa de desarrollo del alienismo, fueron principalmente los médicos de la Casa de Orates los que demandaron un conocimiento específico sobre los nuevos tecnicismos que circularon en el espacio judicial, previo a la formación de especialistas en enfermedades mentales y nerviosas y a la regulación del campo de acción de los médicos de ciudad, futuros médicos legistas. ${ }^{18}$ Así, en los procesos encontramos una multiplicidad de voces nombrando la locura, explicándola de distintas maneras, tratando de entenderla, medirla y pronosticar su evolución. También enfrentamos una variedad de autoridades rastreando la 'normalidad' y diferenciándola de la violencia, de los comportamientos desbordados, de la falta o exceso de afecto y de la exaltación. En esta saturación de voces fueron emergiendo los especialistas, expertos interesados en desplegar su erudición y negociar los límites de la locura en un contexto marcado, como ya indicamos, por las locuras parciales, el intervalo lúcido y el uso de una nueva terminología experta apoyada en los criterios científicos que sostenían la Casa de Locos.

\section{Consideraciones finales}

La pericia alienista en procesos de interdicción proyecta interesantes características del proyecto de medicalización de la locura en la sociedad chilena de mediados del siglo XIX; 
particularidades propias de un cuerpo profesional en formación, marcado por conflictos, necesidades, dudas y ambiciones. La pericia muestra las variables experiencias y estrategias que articularon la profesionalización del ejercicio médico y el posicionamiento de facultativos como testigos expertos en la esfera de la justicia civil. Da cuenta que tanto la validación social de la figura del perito, como el carácter probatorio de sus evaluaciones, fueron moldeadas por una serie de contextos y fuerzas que excedieron el discurso médico y las normativas judiciales.

Observamos primeramente que la formación de una especialización alienista se vinculó al desplazamiento de las evaluaciones periciales desde certificaciones generales a diagnósticos más específicos que consideraron no solo la identificación de ciertas condiciones, sino también su prognosis. Estos diagnósticos, incrementados en el tiempo, ofrecieron la oportunidad de exhibir habilidades profesionales, presentar historias de éxito médico, así como destacar la especificidad del conocimiento experto. Al mismo tiempo estuvieron marcados por el carácter fragmentario y contextual de la 'verdad' médica, emanada desde las mismas desavenencias y contradicciones del cuerpo científico; así también, de la injerencia de hombres y mujeres que, sin mayor conocimiento del discurso médico académico, cuestionaron o validaron, con relativo éxito, dicho saber.

Observamos que la figura del alienista se instaló en el ámbito judicial en relación a otras figuras médicas que, sin poseer un conocimiento experto de la locura, cumplieron un rol central en la evaluación pericial de la demencia. Vemos cómo autoridades de la universidad, profesores extranjeros, médicos de familia, médicos de ciudad, alienistas y expertos en enfermedades mentales y nerviosas, y a fin de siglo, médicos legistas, se encontraron en los juzgados y negociaron su saber. ${ }^{19}$ Los pioneros en el campo del alienismo, como José Ramón Elguero o Manuel Segundo Beca, así como los primeros expertos en enfermedades mentales, como Augusto Orrego Luco, Carlos Sazié o Guillermo del Sol, participaron activamente en procesos de interdicción proyectando un reconocimiento que se incrementó en el transcurso del siglo. Esta legitimación se expresó en el mayor número de alienistas sugeridos por los demandantes o convocados por los jueces, en peritos que se negaban a participar de estos procedimientos por no considerarse capacitados en ese saber y en el debate que concitaban algunas evaluaciones.

Reconocemos, finalmente, un posicionamiento del perito experto en locura en base a su relación con ciertas instituciones. Este proceso fue inaugurado por la formación profesional y el surgimiento de la Casa de Orates y luego complementado por el envío, por parte del gobierno, de los primeros médicos a Europa para su especialización (1874) y la incorporación del curso de enfermedades mentales y nerviosas en la Universidad de Chile (1889). Estos contextos contribuyeron enormemente en la conformación de esa experticia y en su reconocimiento social, influyendo en tanto espacios de formación especializada y de modelación de identidad profesional.

De este modo, la legitimación de la autoridad del perito, en especial del alienista, estuvo estrechamente ligada no solo a la forma y fondo de sus observaciones forenses, al prestigio profesional alcanzado y a su conexión con las instituciones que validaron la nueva ciencia de la locura, sino principalmente al modo en que estas se vincularon con sus propias retóricas de convencimiento y con aquellas desplegadas por demandantes, testigos y demandados. 


\section{NOTAS}

${ }^{1}$ La historiografía sobre medicina legal y pericia médica ha tendido a privilegiar, tanto en Europa como América Latina, el estudio de la esfera penal por sobre la civil. Ver, por ejemplo, Jones (1994); Crawford, Clark (1994); Walker (1968); Eigen (1995); Goold, Kelly (2009); Smith (1981).

${ }^{2}$ Este trabajo se origina de la tesis doctoral realizada entre el 2006 y 2011 en Wellcome Trust Centre for the History of Medicine, University College London, apoyada por WT Doctoral Studentship y UCL ORS.

${ }^{3}$ Considerando juicios de interdicción por demencia del Archivo Nacional de Santiago, los casos de privación de derechos civiles que fueron rotulados como divorcio o administración de bienes (entre otros), el número de interdicciones inscritas en el Registro de Bienes Raíces y las historias citadas en gacetas, revistas, tesinas y manuales es posible confirmar que la incapacitación de dementes fue una práctica altamente extendida en Chile, pudiendo establecerse la existencia de al menos 700 casos de interdicción durante la segunda mitad del siglo XIX.

${ }^{4}$ Hombre o mujer, mayor de 25 años, que pareciera estar sufriendo de una 'demencia habitual', a pesar de comprobarse la existencia de intervalos lúcidos.

${ }^{5}$ Hacia 1850, no encontramos mayores referencias que aludan a las características de la evaluación médica en juicios civiles que abordan la capacidad mental de los locos.

${ }^{6}$ Resulta interesante comprobar que, pese al alcance e implicancias de la interdicción, esta práctica no haya sido mayormente considerada para abordar la historia social de la locura. En los últimos diez años, la incapacidad de dementes ha sido abordada por ejemplo por Houston (2000); Noothens (2007); Suzuki (2006); Sacristán (1999); Di Liscia, Bassa (2003); Di Liscia (2003). Para un estudio de la historiografía latinoamericana centrada en el asilo, ver Sacristán (2009). Agradezco la referencia al trabajo de Delgado (1992), entregada por el/la evaluador/a de este artículo.

${ }^{7}$ Pese a que la historiografía no ha abordado el estudio de la pericia médica en procedimientos civiles, algunos trabajos han dado cuenta de ella como, por ejemplo, Fernández (2000) y Brangier (2008).

${ }^{8}$ Ver, también, la tesis doctoral de Murdock (2000) y Vargas (2001).

${ }^{9}$ Por ejemplo, en materia de salud pública se buscó incrementar el control sobre la población y sobre los médicos en periodos de epidemia a través de la examinación y cuarentena obligatoria que impuso la ley de Policía Sanitaria (1886).

${ }^{10}$ Hacia mediados del siglo XIX, solo existían tres áreas de certificación: medicina y cirugía, química y botánica, y obstetricia.

${ }^{11}$ La Universidad de Chile era la única institución que podía formar y licenciar médicos hasta la creación de la Escuela de Medicina de la Universidad Católica, en 1929, y la de Concepción, en 1924.

${ }^{12}$ Pese a que proyectos de codificación anteriores habían planteado que dicha presencia se requeriría solo "si el juez lo estimare conveniente", como fue propuesto por el proyecto de 1853 (Bello, 1853).

${ }^{13}$ La penalización a los médicos que se negaran a testificar en causas judiciales generó una polémica al interior del cuerpo médico que tensionó por momentos la relación entre ley y medicina. Ver, por ejemplo, Letelier (1877).

${ }^{14}$ Tras el cierre del curso, Elguero fue nombrado profesor de patología interna, mientras el curso fue remplazado por uno sobre enfermedades de niños.

${ }^{15}$ Las pugnas judiciales entre Francisco y Joaquina, las cuales se están estudiando con mayor detalle en mi investigación doctoral, se desarrollaron por varios años y envolvieron la participación de un número significativo de facultativos. La rehabilitación de la interdicción por demencia de Joaquina Aldunate fue decretada por la Corte de Apelaciones de Santiago en 1867.

${ }^{16}$ La monomanía siguió presente en el asilo durante el resto del siglo. En 1885, Manuel Segundo Beca (1885) presentó a la manía como la forma más común de locura en la Casa de Orates $(47,7 \%)$. Las monomanías conformaban solo el 4,3\% del total de internos. Sin embargo, sus fronteras no estaban bien delimitadas y se articulaban constantemente con las manías y las lipemanías. Entre las variantes indicadas por Beca están: monomanía de grandeza o megalomanía, erotomanía, monomanía religiosa o teomanía, monomanía razonante, suicida y cleptomanía. Hacia fines del siglo, encontramos un uso más extendido del término, tanto en expresiones cotidianas que hacían alusión a un desarreglo mental, como en textos de abogados y juristas que intentaban explicar las características de la locura. 
${ }^{17}$ Parte de la atracción historiográfica concitada por Elguero se debe a su calidad de autor de este informe, uno de los primeros reportes que se conocen de un médico de la Casa de Orates. De Elguero no se conocían historias clínicas, ni información sobre sus clases, ni trabajos especializados, salvo el informe publicado en el Ministerio del Interior (Roa, 1992); de ahí que sus evaluaciones judiciales realizadas representen fuentes de gran relevancia para el estudio de las ideas médicas sobre la locura de mediados del siglo XIX.

${ }^{18} \mathrm{Su}$ participación de procesos judiciales fue formalizada por la ley de 1887. Ver "Médicos de Ciudad. Santiago, 31 de diciembre de 1887", en Miranda (1889).

19 Este artículo introduce al quehacer alienista y no aborda el desarrollo de la medicina legal y el posicionamiento de los médicos de ciudad como expertos forenses. En la medida en que el saber alienista se incrementó, surgió una mayor especialización y nuevas ramas que abordaron el estudio de la locura. Nuevas identidades profesionales se relacionaron con la identificación, cuidado o control de las enfermedades mentales como los médicos de ciudad cuya participación judicial fue más notoria en provincia.

\section{REFERENCIAS}

AMUNÁTEGUI, Manuel (Ed.).

Código Civil de la República de Chile. Valparaíso: Imprenta y Librería del Mercurio. 1865.

BECA, Manuel Segundo.

Algo sobre las enfermedades mentales en Chile: recopilación de la estadística de la Casa de Orates, desde su fundación en 1852 hasta la fecha: estudio sobre ella, datos que arroja, deducciones que da a lugar, etc. Anales de la Universidad de Chile, Santiago, p.335-371, ene. 1885.

BELLO, Andrés.

Proyecto de Código Civil. Santiago: Imprenta Chilena. 1853.

BRANGIER, Víctor.

La fragilidad de la simbiosis médico-judicial y la producción de una antropología criminal: juzgados del crimen de Santiago, 1874-1906. Tesis (Maestría) - Facultad de Filosofía y Humanidades, Universidad de Chile, Santiago. 2008.

CORREA GÓMEZ, María José.

Lay people, medical experts and mental disorders: the medicalization of insanity through the incapacitation of the mentally ill, 1830-1925. Tesis (Doctorado) - Centre for the History of Medicine, University College London, London. 2012.

CORREA GÓMEZ, María José.

Violencias ejercidas en los cuerpos enajenados: encierro terapéutico y privación de derechos civiles, Chile central (1850-1870). Nuevo Mundo Mundos Nuevos, Paris, Debates. 2009. Disponible en: http://nuevomundo.revues.org/57798.

Acceso en: 21 mayo 2013. 2009.

COSTA, Claudio.

Don Diego Portales y el caso Paddock. Revista Médica de Chile, Santiago, v.112, p.842-848. 1984.

CRAWFORD, Catherine.

Medicine and the law. In: Bynum, William;
Porter, Roy. Companion encyclopedia of the history of medicine. London: Routledge. 1993.

CRAWFORD, Catherine; CLARK, Michael (Eds.). Legal medicine in history. Cambridge: Cambridge University Press. 1994.

DE LA CRUZ, Ernesto (Ed.).

Epistolario de Don Diego Portales, 1821-1837,

II. Santiago: Imprenta Dirección General de Prisiones. 1937.

DE LA PLATA, Elías.

Interdicción por demencia. Santiago: Imprenta de la Unión. 1887.

DEL REAL, José Luis.

Curaduría de mayores incapaces. Santiago: Imprenta Universitaria. 1903.

DELGADO, Pedro.

As razões da tutela: psiquiatria, justiça e cidadania do louco no Brasil. Rio de Janeiro: Té Cora. 1992.

DI LISCIA, Silvia.

Mujeres, locura e incapacidad civil en Argentina, 1890-1920. Aljaba, Segunda Época, Revista de Estudios de la Mujer, v.8, p.89-105. 2003.

DI LISCIA, Silvia; BASSA, Daniela.

Médicos, jueces y locos: sobre peritaje de insania y justicia en el interior argentino, 1890-1930.

Horizontes, Bragança Paulista, v.21, p.15-26. 2003.

EIGEN, Joel Peter.

Witnessing insanity: madness and mad-doctors in the English court. New Haven, CT: Yale University Press. 1995.

ELGUERO, Ramón.

Informe del médico de la casa de locos: memoria que el ministro de estado en el departamento del interior presenta al Congreso Nacional de 1863. Santiago: Imprenta Nacional. 1863.

FERNÁNDEZ, Marcos.

La explicación y sus fantasmas: representaciones del delito y de la eximición de responsabilidad 
penal en el Chile del siglo XIX. Revista de Historia Social y de las Mentalidades, Santiago, v.1, n.4, p.105-130. 2000.

FOUCAULT, Michel.

Abnormal: lectures at the Collège de France, 1974-1975. New York: Picador. 2003.

GOLDSTEIN, Jan.

Console and classify: the French psychiatric profession in the nineteenth century. Chicago: The University of Chicago Press. 2001.

GOOLD, Imogen; KELLY, Katherine.

Lawyers' medicine: the legislature, the courts and medical practice, 1760-2000. Oregon: Hart Publishing. 2009.

HOUSTON, Robert.

Madness and society in eighteenth-century Scotland. Oxford: Clarendon Press. 2000.

\section{INTERDICCIÓN.}

Interdicción. Archivo Histórico Nacional de Chile, Fondo Judicial de Santiago, L 20 A, p.10. 1862.

JONES, Carol.

Expert witnesses: science, medicine and the practice of law. Oxford: Clarendon Press. 1994.

LETELIER, Sandalio.

El código penal y la profesión de médicocirujano. Revista Chilena, Santiago, v.7. 1877.

MIRANDA, Roberto.

Disposiciones vigentes en Chile sobre policía sanitaria y beneficencia pública. Santiago: Roberto Miranda Editor. 1889.

MORRELL, Jack.

Professionalization. In: Olby, Robert et al.

Companion to the history of modern science.

London: Routledge. 1996.

MURDOCK, Carl.

Strategic mercies: physicians, the state and public health in Chile, 1875-1910. Tesis (Doctorado) University of Connecticut. 2000.

\section{MURDOCK, Carl.}

Physicians, the state and public health in Chile, 1881-1891. Journal of Latin American Studies, v.27, n.3, p.551-567. 1995.

NOOTHENS, Thierry.

Fous, prodigues et ivrognes: familles et deviance a Montréal au XIXe siècle. Québec: McGill-Queen's University Press. 2007.

PUGA BORNE, Federico.

Compendio de medicina legal adaptado a la legislación chilena. Santiago: Imprenta Cervantes. 1896.

ROA, Armando.

Augusto Orrego Luco en la cultura y la medicina chilena. Santiago: Editorial Universitaria. 1992.
SACRISTÁN, Cristina.

La locura se topa con el manicomio: una historia por contar. Cuicuilco, v.16, n.45, p.163-189. 2009.

SACRISTÁN, Cristina.

Locura y justicia en México: la psiquiatría, la familia y el individuo frente a la modernidad liberal: el caso Raygosa (1873-1877). Tesis (Doctorado) - Departamento de Antropología, Filosofía y Trabajo Social, Universitat Rovira i Virgili, Tarragona. 1999.

SCHNEIDER, Germán.

El rol del médico. Revista Médica, v.1, n.3, p.121-123. 1872.

SERRANO, Sol.

Universidad y nación: Chile en el siglo XIX. Santiago: Editorial Universitaria. 1994.

SMITH, Roger.

Trial by medicine: insanity and responsibility in Victorian trials. Edinburgh: Edinburgh University Press. 1981.

STABILI, María Rosaria.

Jueces y justicia en el Chile liberal.

In: Carmagnani, Marcelo (Comp.).

Constitucionalismo y orden liberal: América Latina, 1850-1920. Turín: Otto Editore. 2000.

SUZUKI, Akihito.

Madness at home: the psychiatrist, the patient and the family in England 1820-1860. Berkeley: University of California Press. 2006.

TORO, David; ECHEVERRÍA, David.

Código de procedimiento civil. Santiago: Imprenta Barcelona, 1902.

VARGAS, Juan Eduardo.

Rasgos de la autoimagen social y profesional de los médicos (1872-1925). Ars Medica, Santiago, v.4, n.4, p.103-126. 2001.

VERA, Robustiano.

Prontuario de enjuiciamiento criminal. Santiago: Imprenta Victoria. 1884.

VILA, Bernadino.

Prontuario de los juicios 1. Santiago: Imprenta de la Sociedad. 1857.

WALKER, Nigel.

Crime and insanity in England VI. Edinburgh: Edinburgh University Press. 1968.

WILLIAMS, Melanie.

Medico-legal stories of female insanity: three nullity suits. Feminist Legal Studies, v.1, n.1, p.3-31. 1998.

WRIGHT, David.

The certification of insanity in nineteenthcentury in England and Wales. History of Psychiatry, v.9, n.35, p.267-290. 1998. 